\title{
The LPI Performance Analysis of Chaos-MCPC Radar Signals based on Cyclic Autocorrelation Accumulation factor
}

\author{
Yang $\mathrm{Li}^{1,}$, , Songhua $\mathrm{He}^{2}$, b , Fubin Chen², Jianping $\mathrm{Ou}^{2}$ and Jun Zhang ${ }^{2}$ \\ ${ }^{1}$ School of Information Science and Engineering, Hunan University, Changsha 410082, China; \\ ${ }^{2}$ School of Electronic Science and Engineering, National University of Defense Technology, Changsha \\ 410073, China. \\ a2292621826@qq.com, b13973132618@139.com
}

Keywords: Cyclic autocorrelation, radar signals, MCPC, LPI.

\begin{abstract}
In this paper, for the first time, from the perspective of intercept receiver, based on the cyclic autocorrelation accumulation factor ( $U$ value), the low probability of intercept (LPI) performance of several traditional radar signals, COCS P4-MCPC radar signal and Chaos-MCPC radar signal are evaluated by simulation with some quantitative analyses. The simulation results show that when the reception SNR is low, regardless of low or high repetition frequency, the Chaos-MCPC signal possesses the best LPI performance.
\end{abstract}

\section{Introduction}

With the development of electronic countermeasure (ECM) and reconnaissance technique, the electromagnetic environment become more and more complex. In order to enhance the viability of the radar, in 1980s, Schleher firstly proposed the theory of low probability of intercept (LPI) radar, and put forward the concept of intercept factor based on LPI radar equation [1]. Because LPI radar can be used for avoiding the signal interception of the third-party reconnaissance receiver when tracking target, LPI radar has been extensively used in military activity.

However, in the past two decades, with the advancement of the interception technique, the subsistence of the traditional LPI radar become being challenged. In order to further enhance the viability of the LPI radar, a lot of researchers have begun to study the design of LPI radar signal. For example, after orthogonal frequency-division multiplexing (OFDM) was introduced into radar system by Jankiraman et al. in 1998 [2], a lot of research work about the design of LPI signals based on OFDM have been carried out and a lot of LPI signal based on OFDM have been proposed. One of the representative LPI signals is the MCPC signal which was proposed by professor Levanon in 2000 for the first time [3]. Recently, many scholars at home and abroad reported that the MCPC signal has a better LPI performance than traditional LPI signal [4, 5, 6, and 7]. Nevertheless, the analyses of the LPI performance of the MCPC signal are generally based on its ambiguity function [8], while the quantitative analyses of the LPI performance of the MCPC signal are lacked. In order to realize the quantitative analysis of the performance of the LPI signal, a method based on cyclic autocorrelation accumulation factor (U value) was proposed by our previous work [9].

In this paper, based on U value, we evaluate the LPI performance of two MCPC signals including the chaotic binary phase code based MCPC signal (chaos-MCPC) and P4 cyclic shift code based MCPC (COCS P4-MCPC). In the simulation, a comparison is made between the LPI performance of the MCPC signals and the LPI performance of the normal LPI signals. The simulation results show that the MCPC signals possess a better LPI performance than the normal LPI signals. Meanwhile, it is also demonstrated that the LPI performance of the chaos-MCPC is better than that of the COCS P4-MCPC. To the best of our knowledge, our work is the first time to verify the LPI performance of the chaos-MCPC in a way of quantitative analysis. 


\section{Cyclic Autocorrelation Accumulation Factor (U value)}

Cyclic autocorrelation method is an effective method to detect and intercept the periodic radar signals. It can be used in interception receiver for assisting the interception behavior. And it also can be used for analyzing the LPI performance of different radar signal. In our previous work [9], based on the cyclic autocorrelation method, the $U$ value is proposed for evaluating the LPI performance of radar signal. The $U$ value can be obtained as the following processes.

Firstly, according to the equation of radar detection and reconnaissance range [1], the LPI factor can be obtained and it has a special relationship with the processing gain of radar receiver and reconnaissance receiver as the following equation:

$$
\alpha \propto\left(\frac{D}{D_{r}}\right)^{\frac{1}{4}}
$$

Where $\alpha$ is the LPI factor, $D_{i}$ is the processing gain of the reconnaissance receiver and $D_{r}$ is the processing gain of the primordial radar receiver.

According to Eq. (1), when there are several different signals which are with the same time-bandwidth, average power, and processing gain of the radar receiver $D_{r}$, one of these signals which has a lower processing gain of reconnaissance receiver, will be with a better anti-interception performance. Hence, the LPI performance of these signals can be evaluated by using the processing gain of reconnaissance receiver instead of the LPI factor.

Assumed that the thermal noise of the reconnaissance receiver is Gaussian white noise, and the signal-to-noise ratio (SNR) is far less than $0 \mathrm{~dB}$, by using the analytical method based on cyclic autocorrelation and according to the analysis method proposed in Ref. [9], the gain of the reconnaissance receiver can be obtained as

$$
D_{i}(\mathrm{~m})=\frac{\sqrt{N_{3}} N_{2}\left|\sum_{n=m}^{N_{2}-m-1} s(n+m) s^{*}(n-m) e^{-j \frac{2 \pi}{N_{1}} n}\right|}{\sqrt{N_{1}[1+\delta(m)]} \sum_{n=0}^{N_{2}-1}|s(n)|^{2}}
$$

where $s(n)$ is the radar signal, $N_{1}$ is the signal period, $N_{2}$ is the width of one pulse in the signal, $N_{3}$ is the amount of the pulses in the signal, $\delta()$ is the unit pulse function, and $m\left(0 \leqslant m<N_{2} / 2\right)$ is the time delay for the cyclic autocorrelation.

Obviously, as derived in Ref. [9], when $N_{3}$ is a specific number, when using Di to evaluate the LPI performance, it is equivalent to use the following equation:

$$
U=\max \left\{\left.\frac{1}{\sqrt{2 N_{1}}}\left|\sum_{n=0}^{N_{2}-1}\right| s(n)\right|^{2} e^{-j \frac{2 \pi}{N_{1}} n}\left|, \max _{m \in\left[1, N_{2} / 2\right)} \frac{1}{\sqrt{N_{1}}}\right| \sum_{n=m}^{N_{2}-m-1} s(n+m) s^{*}(n-m) e^{-j \frac{2 \pi}{N_{1}} n} \mid\right\}
$$

Where $U$ is the cyclic autocorrelation accumulation factor. And according to Eq. (1) and Eq. (3), it can be found that when the $U$ value of a radar signal is smaller, the anti-interception performance of the signal is better.

\section{Multi-carrier Phase Code (MCPC) Pulses Signal}

By introducing the phase encoding technique into OFDM, the MCPC pulses signal was proposed in 2000 [3]. As we know, in general, the MCPC pulses signal can be uniformly expressed as

$$
s(\mathrm{t})=\sum_{n=1}^{N} \sum_{m=1}^{M} \omega_{n} a_{n, m} \mathrm{u}\left[t-(\mathrm{m}-1) \mathrm{t}_{b}\right] \exp \left[\mathrm{j} 2 \pi\left(\mathrm{n}-\frac{N+1}{2}\right) \Delta f t\right], u(\mathrm{t})=\left\{\begin{array}{l}
1,0 \leq t<t_{b} \\
0, \text { otherwise }
\end{array}\right.
$$

Where $N$ is the subcarrier number and $M$ is the amount of the phase codes modulated on each carrier, $a_{n, m}$ is the $m$-th element of the phase code sequence modulated on $n$-th carrier and $t_{b}$ is the unit code 
duration, the frequency difference between two adjacent carriers is $\Delta f=1 / t_{b}$, and the complex weight associated with the $n$-th carrier is $\omega_{n}=\left|\omega_{n}\right| e^{j \phi_{n}}\left(\sum_{n=0}^{N-1}\left|\omega_{n}\right|^{2}=1\right)$.

At present, based on different phase code schemes, different kind of encoded MCPC signals have been proposed. For example, based on all cyclic shifts of P4 code and chaotic code, COCS P4-MCPC [3] and chaos-MCPC [7] have been proposed, respectively. In this paper, the two kinds of MCPC signal are mainly studied.

\subsection{COCS P4-MCPC.}

P4 code is a kind of poly-phase code with ideal periodic autocorrelation property. Its phase sequence is defined as

$$
\phi_{m}=\frac{2 \pi}{M}(\mathrm{~m}-1)\left(\frac{m-1-M}{2}\right)
$$

Where $M$ is the code amount, $1 \leqslant m<M$. After P4 code is used in MCPC, by shifting the P4 code in a consecutive cyclic way for encoding the subcarriers of the MCPC signal, the COCS P4-MCPC can be obtained as

$$
s(t)=\sum_{n=1}^{N} \sum_{m=1}^{M} w_{n} a_{\bmod (n+m-1, M)} u\left(t-(m-1) t_{b}\right) \exp \left[j 2 \pi\left(\mathrm{n}-\frac{N+1}{2}\right) \Delta f t\right]
$$

Where $a_{\bmod (n+m-1, M)}$ is the $m$-th element of the sequence modulated on the $n$-th carrier and it equals to

\subsection{Chaos-MCPC.}

$$
a_{\bmod (n+m-1, M)}=\exp \left(j \phi_{\bmod (n+m-1, M)}\right)
$$

Because the chaotic sequence is of good controllability and can be simply generated with unlimited length, the chaotic sequence is been widely used in the field of radar signal processing. It can be used in LPI radar system. And based on chaotic sequence and specific mapping scheme, chaos-MCPC radar signal can be obtained [7]. In this paper, based on Bernulli mapping scheme, the binary phase chaotic coded MCPC is investigated. In our scheme, the chaos-MCPC signal can be expressed as

$$
s(t)=\sum_{n=1}^{N} \sum_{m=1}^{1} w_{n} \exp \left(j \theta_{n}\right) u\left(t-(m-1) t_{b}\right) \exp \left[j 2 \pi\left(\mathrm{n}-\frac{N+1}{2}\right) \Delta f t\right]
$$

Where $\theta_{n}$ is the phase sequence of the chaotic code in our schem(\&) which is defined as

$$
\theta_{n}=2 \pi \cdot \operatorname{ceil}\left(2 \cdot x_{n}+0.5\right) / 2
$$

In Eq. (9), $x_{n}$ can be obtained from the following iterative equation

$$
x_{n+1}=\left\{\begin{array}{l}
b x_{n}+0.5, x_{n}<0 \\
b x_{n}-0.5, x_{n} \geq 0
\end{array}, b=2-\varepsilon, x_{n} \in[-0.5,0.5]\right.
$$

Where $\varepsilon>0$, and the initial value of the equation is $x_{0} \in[-0.5,0.5]$.In the subsequent simulation, $b$ is set at $1.995, x_{0}$ is set at 0.25 . Although it is believed that Chaos-MCPC signal is of better LPI performance than the normal LPI signals such as BPSK, PN-OFDM, etc., quantitative analyses for the LPI performance comparison between Chaos-MCPC and normal LPI signals are lacked.

\section{Simulation Setup and Results}

In the MATLAB-based simulation, when the simulation system and condition is set as the same with the simulation system and condition in Ref. [9], e.g., the reception SNR is also set at $0 \mathrm{~dB}$, a comparison is made between the two MCPC signals and the traditional LPI radar signals including BPSK, PN-OFDM and SCAPW-OFDM signals. In order to make a fair comparison, some key parameters of these signals are set at the same constant. For example, the pulse width $N_{2}$ of these 

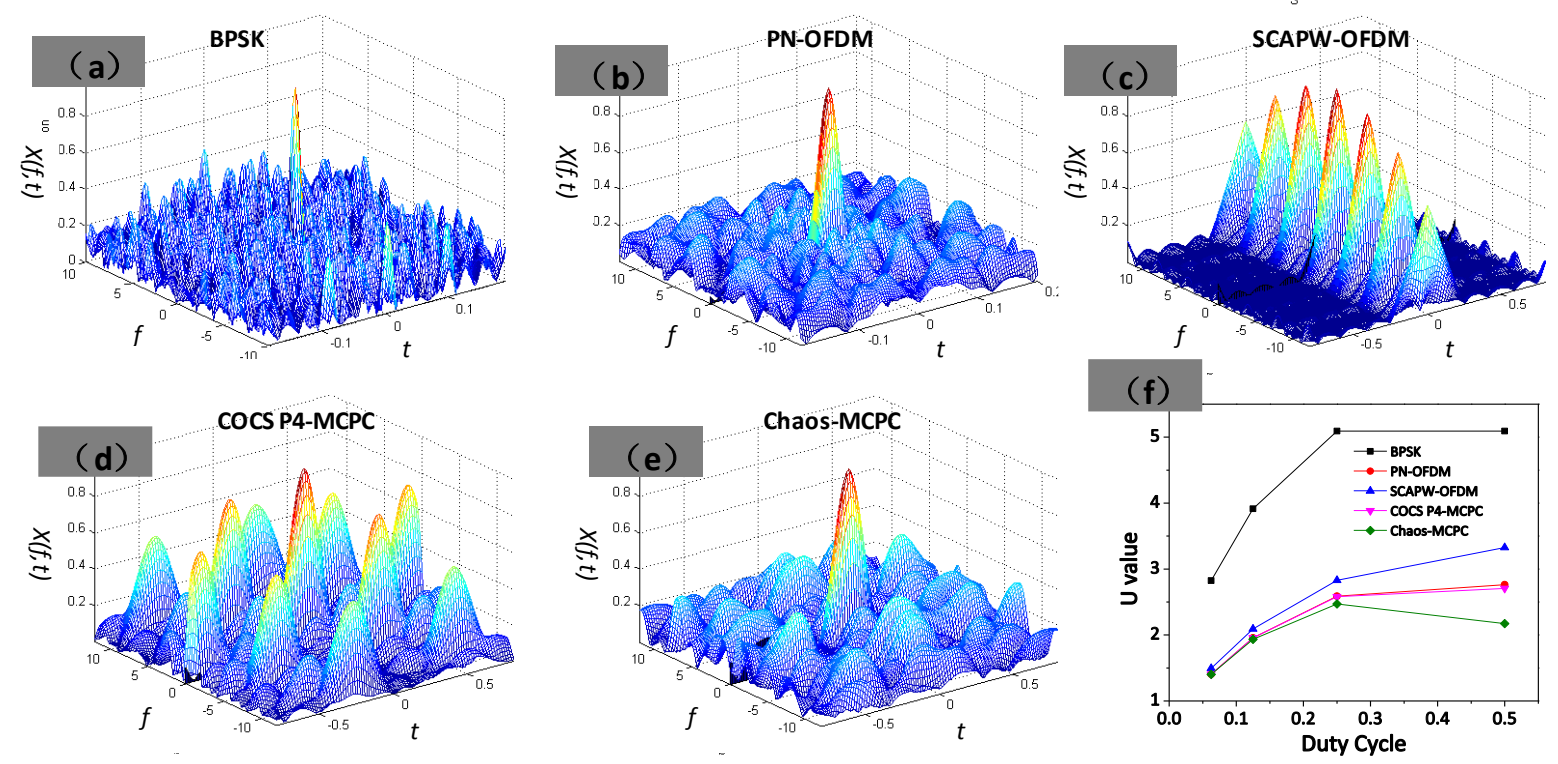

Fig. 1. The results of the simulation

signals are set at 256 , the averaging power $E$ of these signals are set at 256 , and the time-bandwidth product of these signals are set at 32 , etc.

Firstly, the ambiguity functions of these signals are simulated and demonstrated in Fig. 1 (a) (e). It is reported by some previous researches [8] that LPI performance of a radar signal can be reflected from its own ambiguity function to some extent. However, as shown in Fig. 1 (a) (e), it is difficult to distinguish the LPI performance of these signals so that the LPI performance comparison is difficult to be realized.

Secondly, by varying the period $N_{l}$ of these signals in $(512,1024,2048,4096)$, i.e., the duty cycle of these signals is set at $1 / 2$ or $1 / 4$ or $1 / 8$ or $1 / 16$, the $U$ value of these signals are calculated. Fig. 1 (f) shows the curves of the $U$ value versus duty cycle of these signals. Obviously, the LPI performance of the Chaos-MCPC signal is better than that of the other signals. And when the value of the duty cycle is higher, the performance advantage of the Chaos-MCPC signal become more obvious. Most importantly, it can be found that no matter when the value of the duty cycle is low or high, i.e., regardless of low or high repetition frequency, the chaos-MCPC signal possesses the best LPI performance and the LPI performance of the MCPC signal is always better than that of the other signals. This quantized result further verifies that phase coding can be used in OFDM for effectively enhancing the LPI performance.

\section{Conclusion}

In this paper, by using $U$ value, through numerical simulation, for the first time, the LPI performance of BPSK, PN-OFDM, SCAPW-OFDM and two kinds of MCPC including COCS P4-MCPC and Chaos-MCPC are compared. The simulation results show that the LPI performance of the MCPC signals are greatly superior to the traditional signals such as BPSK and PN-OFDM signals. Most importantly, the results also verify the superiority of the LPI performance of the Chaos-MCPC signal.

\section{References}

[1]. D.C.Schleher. Low Probability of Intercept Radar. Radar Conference. 1985, p. 346-349.

[2]. Jankiraman M, Wessels B J, Genderen P V. System design and verification of the PANDORA multifrequency radar. Proceedings of 5th International Conference on Radar Systems. Brest, France, May.1999, p.17-21. 
[3]. N. Levanon. Multi-frequency complementary phase-coded radar signal, IEE Proceedings: Radar, Sonar and Navigation, Vol.147 (2000) No.6, p. 276-284.

[4]. Mozeson E, Levanon N. Multicarrier radar signals with low peak-to-mean envelope power ratio. IEE Proceedings: Radar, Sonar and Navigation, Vol.150 (2003) No.2, p. 71-77.

[5]. Q.Huang, Li Y, Y. Zeng, et al. Design and characteristic analysis of multicarrier chaotic phase coded radar pulse train signal. International Journal of Antennas and Propagation, 2014, pp.1-8.

[6]. DENG Bin, WEIXi-zhang, LI Xiang. Parameter Designing of Random Shifted Phase-coded MCPC Radar Signal. Journal of national university of design technology, Vol.33 (2011) No.2, p. 68-72.

[7]. Huo Kai and Zhao Jingjing. A design method of four-phase-coded OFDM radar signal based on Bernoulli chaos. Journal of Radars, Vol.5 (2016) No.4, p. 361-372.

[8]. A. H. Xu: Study on low probability of intercept radar system based on OFDM (Ph. D dissertation, Nanjing University of Science and Technology, China, 2012).p.24.

[9]. Wang Xia, He Song hua, Oujian Ping, et al. The LPI performance analysis and comparison of radar signals by cyclic autocorrelation method. Signal Processing, Vol.31 (2015) No.3, p.328-335. 